\title{
Population III Gamma-Ray Burst
}

\section{Kunihito Ioka, Yudai Suwa, Hiroki Nagakura, Rafael S. de Souza, and Naoki Yoshida}

KEK Theory Center, 1-1 Oho, Tsukuba 305-0801, Japan

email: kunihito.ioka@kek.jp

\begin{abstract}
Gamma-ray bursts (GRBs) are unique probes of the first generation (Pop III) stars. We show that a relativistic gamma-ray burst (GRB) jet can potentially pierce the envelope of a very massive Pop III star even if the Pop III star has a supergiant hydrogen envelope without mass loss, thanks to the long-lived powerful accretion of the envelope itself. While the Pop III GRB is estimated to be energetic $\left(E_{\gamma, \text { iso }} \sim 10^{55} \mathrm{erg}\right)$, the supergiant envelope hides the initial bright phase in the cocoon component, leading to a GRB with a long duration $\sim 1000(1+z) \mathrm{s}$ and an ordinary isotropic luminosity $\sim 10^{52} \mathrm{erg} \mathrm{s}^{-1}\left(\sim 10^{-9} \mathrm{erg} \mathrm{cm}^{-2} \mathrm{~s}^{-1}\right.$ at redshift $\left.z \sim 20\right)$, although these quantities are found to be sensitive to the core and envelope mass. We also show that Pop III.2 GRBs (which are primordial but affected by radiation from other stars) occur $>100$ times more frequently than Pop III.1 GRBs, and thus should be suitable targets for future $\mathrm{X}$-ray and radio missions. The radio transient surveys are already constraining the Pop III GRB rate and promising in the future.
\end{abstract}

Keywords. gamma rays: bursts, stars: Population III

My talk is mainly based on Suwa \& Ioka (2011), Nagakura, Suwa \& Ioka (2011), de Souza, Yoshida \& Ioka (2011), Ioka \& Mészáros (2005), Ioka (2003).

\section{Introduction}

The ancient era of the first generation stars (Population III; Pop III) - the end of the dark age - is still an unexplored frontier in the modern cosmology. Gamma-Ray Bursts (GRBs) are potentially powerful probes of the Pop III era. The highest redshift of GRB is increasing rapidly, almost approaching $z=10$ (GRB 050904 at $z=6.3$, GRB 080913 at $z=6.7$, GRB 090423 at $z=8.2$, GRB 090429B at $z=9.4$ ). The GRBs are presumed to manifest the gravitational collapse of a massive star - a collapsar - to a black hole with an accretion disk, launching a collimated outflow (jet) with a relativistic speed. The massive stars quickly die within the Pop III era. The GRBs, the most luminous objects in the Universe, are detectable in principle out to redshifts $z \sim 100$, while their afterglows are observable up to $z \sim 30$ (Ioka \& Mészáros 2005, Ioka 2003).

The first stars are predicted to be predominantly very massive $\gtrsim 100 M_{\odot}$ (Abel, Bryan \& Norman 2002, Bromm, Coppi, \& Larson 2002). The central part collapses first to a tiny $\left(\sim 0.01 M_{\odot}\right)$ protostar, followed by the rapid accretion of the surrounding matter to form a massive first star (Omukai \& Palla 2003, Yoshida, Omukai, \& Hernquist 2008). The stars with $140-260 M_{\odot}$ are expected to undergo the pair-instability supernovae without leaving any compact remnant behind, while those above $\sim 260 M_{\odot}$ would collapse to a massive $\left(\sim 100 M_{\odot}\right)$ black hole with an accretion disk, potentially leading to scaled-up collapsar GRBs (Komissarov \& Barkov 2010, Mészáros \& Rees 2010, Suwa \& Ioka 2011, Nagakura, Suwa \& Ioka 2011). The Pop III GRB rate would be rare $\sim 0.1-10 \mathrm{yr}^{-1} \mathrm{but}$ within reach (de Souza, Yoshida \& Ioka 2011). These GRBs also mark the formation of the first black holes, which may grow to supermassive black holes (BHs) via merger or accretion. 
However, the zero-metal stars could have little mass loss by the line driven wind, and thereby have a large $\left(R_{*} \sim 10^{13} \mathrm{~cm}\right)$ hydrogen envelope at the end of life (red supergiant (RSG) phase). Especially for Pop III stars, the mass accretion continues during the main sequence phase, so that the chemically homogeneous evolution induced by rapid rotation might not work (Ohkubo et al. 2009). Their extended envelopes may suppress the emergence of relativistic jets out of their surface even if such jets were produced (Matzner 2003). The observed burst duration $T \sim 100 \mathrm{~s}$, providing an estimate for the lifetime of the central engine, suggests that the jet can only travel a distance of $\sim c T \sim 10^{12} \mathrm{~cm}$ before being slowed down to a nonrelativistic speed. This picture is also supported by the nondetections of GRBs associated with type II supernovae. Nevertheless, this may not apply to the Pop III GRBs because the massive stellar accretion could enhance the jet luminosity and duration and therefore enable the jet to break out the first stars.

We discuss the jet propagation in the first stars using the Pop III stellar structure to estimate the jet luminosity via accretion and to predict the observational main characters of the Pop III GRBs, such as energy and duration. The stellar structure also determines the jet head velocity inside the star. We treat both the jet luminosity and its penetrability with the same stellar structure consistently for the first time.

\section{Progenitor structure and jet propagation}

We employ three representative progenitors in Fig. 1. Red line shows the density profile of Pop III star with $915 M_{\odot}$ (model Y-1 of Ohkubo et al. 2009). Blue indicates the GRB progenitor with $16 M_{\odot}$. Green line represents the progenitor of ordinary core-collapse supernovae with $15 M_{\odot}$. The density profiles are roughly divided into two parts: core and envelope. The GRB progenitor (WR star) does not have hydrogen envelope, while Pop III and RSG keep their envelope so that these stars experience the envelope expansion triggered by core shrinkage after the main sequence.

We can calculate the accretion rate, $\dot{M}$, using these density profiles. The accretion timescale of matter at a radius $r$ to fall to the center of the star is roughly equal to the free-fall timescale, $t_{f f} \approx \sqrt{r^{3} / G M_{r}}$. Then we can evaluate the accretion rate at the

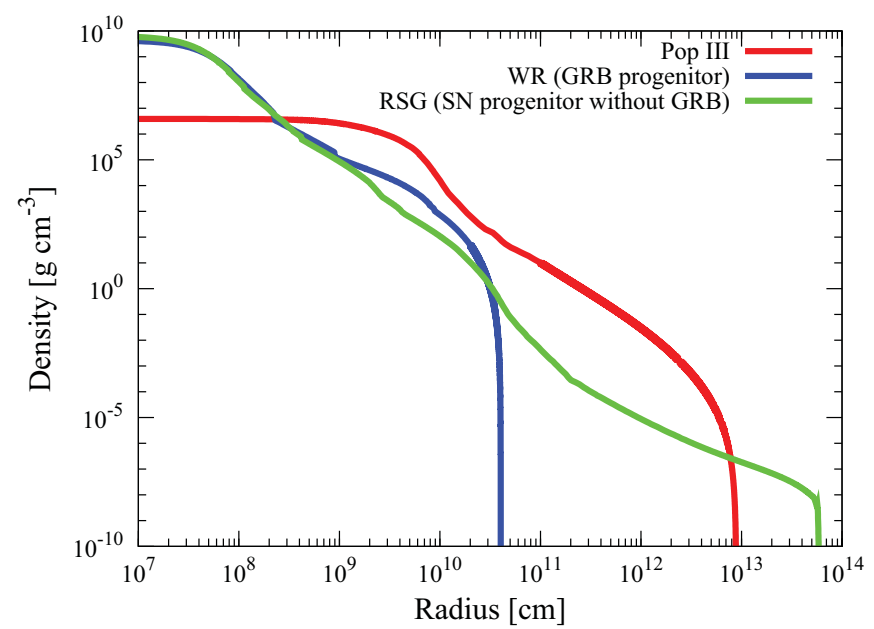

Figure 1. Density profiles of investigated models. Red, blue, and green lines correspond to Pop III star $\left(M=915 M_{\odot}\right)$, Walf-Rayet star (WR; GRB progenitor, $M=16 M_{\odot}$ ), and red supergiant (RSG; SN progenitor without GRB, $M=15 M_{\odot}$ ), respectively. Pop III and RSG have a hydrogen envelope, which expands to a large radius, while WR has only a core. 
center as $\dot{M}=d M_{r} / d t_{f f}$. The rotation law inside the star as well as the jet production mechanism are unknown so that we introduce an efficiency parameter to connect the (free-fall) mass accretion rate and jet luminosity, $\eta=L_{j} / \dot{M} c^{2}$, which will be normalized by the observed GRBs.

The jet head velocity $c \beta_{h}$ inside the star is determined by the pressure balance between the jet $\left(\sim L_{j} / \pi \theta_{j}^{2} r^{2} c\right)$ and the envelope $\left(\sim \rho c^{2} \beta_{h}^{2}\right)$. The shocked jet and shocked envelope go sideways and become the cocoon component (Matzner 2003, Ioka et al. 2011).

Figure 2 shows our results. For the Pop III star, the core accretion ends at a few seconds, which is hidden by the stellar envelope. However the envelope accretion still continues and the jet finally breaks out the stellar envelope. Therefore we conclude that the GRB jet can break out the Pop III first star. The key is the envelope accretion that can enable the jet breakout from the envelope itself. Note that we confirm that the jet cannot break out of a RSG, which is consistent with the nondetections of GRBs associated with type II supernovae.

\section{Characteristic of Pop III GRB}

From Fig. 2, we can find that the duration of Pop III GRB at redshift $z$ is

$$
T_{\mathrm{GRB}}=T_{90}(1+z) \approx 30000 \mathrm{~s}\left(\frac{1+z}{20}\right),
$$

which is much longer than the canonical duration of GRBs, $\sim 20 \mathrm{~s}$. The total isotropicequivalent energy of Pop III GRB is

$$
E_{\gamma, \text { iso }}=\varepsilon_{\gamma} E_{\text {iso }} \approx 1.2 \times 10^{55}\left(\frac{\varepsilon_{\gamma}}{0.1}\right) \mathrm{erg},
$$

where $\varepsilon_{\gamma}$ is the conversion efficiency from the jet kinetic energy to gamma rays. It should be noted that this value is comparable to the largest $E_{\gamma \text {,iso }}$ ever observed, $\approx 9 \times 10^{54} \mathrm{erg}$

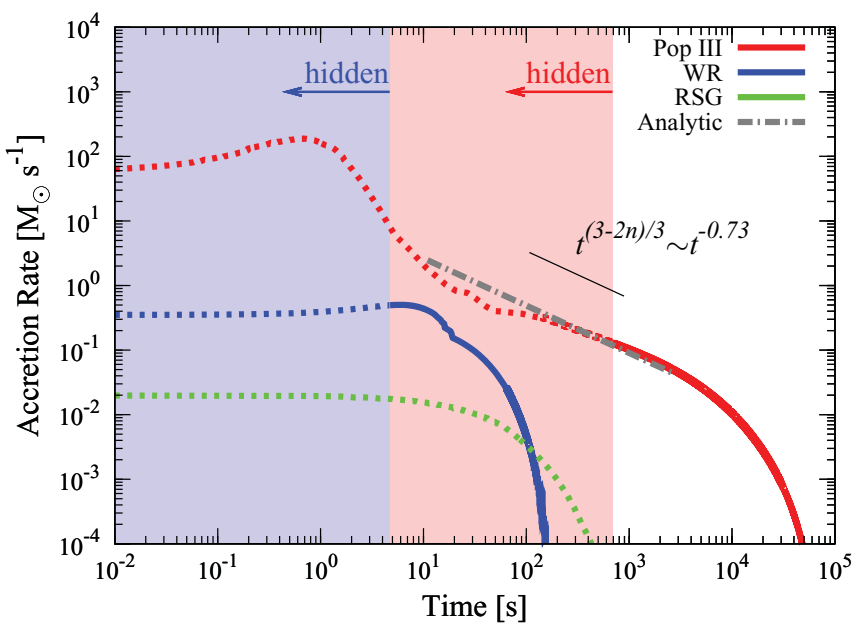

Figure 2. Accretion rates as a function of time. Red, blue, and green lines show Pop III, WR, and RSG, respectively. Dotted regions represent the jet propagating inside the star, while the solid regions correspond to the time after the jet breakout. Solid lines give information of observables (e.g., duration and energetics of GRB). On the other hand, dotted regions show the hidden energy inside the star that goes into the nonrelativistic cocoon component. The gray dot-dashed line represents the analytic model. 
for GRB 080916C. Since the large isotropic energy is stretched over the long duration, the expected flux just after the breakout is not so bright,

$$
F=\frac{\varepsilon_{\gamma} L_{\text {iso }}}{4 \pi r_{L}^{2}} \sim 10^{-9} \mathrm{erg} \mathrm{cm}^{-2} \mathrm{~s}^{-1},
$$

which is smaller than the Swift Burst Array Telescope (BAT) sensitivity, $\sim 10^{-8}$ erg $\mathrm{cm}^{-2} \mathrm{~s}^{-1}$. However, there must be a large variety of the luminosity as ordinary GRBs so that more luminous but rare events might be observable by BAT.

The above discussions strongly depend on the envelope mass because the stellar radius is highly sensitive to the envelope mass. We derive analytical dependences on the model parameters in Suwa \& Ioka (2011), Nagakura, Suwa \& Ioka (2011). The matter entrainment from the envelope is also crucial for the fireball dynamics and the GRB spectra (Ioka 2010). Since the envelope of the Pop III star is different from that of present-day stars, the GRB appearance is also likely distinct from the observed ones (Ioka et al. 2011). These are interesting future problems.

The mass of Pop III star could be much smaller than $\sim 1000 M_{\odot}$ down to $\sim 40 M_{\odot}$ if we consider the photoevaporation of the accretion disk, the disk fragmentation, or the Pop III star formation from once-ionized gas (i.e., Pop III.2 star). The jet breakout is also possible for these light Pop III stars as shown by Nagakura, Suwa \& Ioka (2011).

We also calculate the Pop III GRB rate, and find that Pop III.2 GRBs (which are primordial but affected by radiation from other stars) occur $>100$ times more frequently than Pop III.1 GRBs, and thus should be suitable targets for future GRB missions. The radio afterglows of Pop III GRBs are observable up to $z \sim 30$ (Ioka \& Mészáros 2005, Ioka 2003). Interestingly, the radio transient searches are already constraining the Pop III GRB rate. Future surveys by EVLA, LOFAR and SKA are promising.

\section{References}

Abel, T., Bryan, G. L., \& Norman, M. L. 2002 Science, 295, 93

Bromm, V., Coppi, P. S., \& Larson, R. B. 2002 ApJ, 564, 23

de Souza, R. S., Yoshida, N., \& Ioka, K. 2011 A\&A, 533, A32

Ioka, K. 2003, ApJ, 598, L79

Ioka, K. \& Mészáros, P. 2005 ApJ, 619, 684

Ioka, K. 2010 Prog. Theor. Phys., 124, 667

Ioka, K., Ohira, Y., Kawakana, N., \& Mizuta, A. 2011 Prog. Theor. Phys., 126, 555

Komissarov, S. S., \& Barkov, M. V. 2010 MNRAS, 402, L25

Matzner, C. D. 2003 MNRAS, 345, 575

Mészáros, P. \& Rees, M. J. 2010 Apj, 715, 967

Nagakura, H., Suwa, Y., \& Ioka, K. 2011 arXiv:1104.5691

Ohkubo, T., Nomoto, K., Umeda, H., Yoshida, N., \& Tsuruta, S. 2009 Apj, 706, 1184

Omukai, K., \& Palla, F. 2003 Apj, 589, 677

Suwa, Y., \& Ioka, K. 2011 ApJ, 726, 107

Yoshida, N., Omukai, K., \& Hernquist, L. 2008 Science, 321, 669

\section{Discussion}

Question: How about a binary Pop III GRB?

Kuninito IokA: We study a GRB from a single Pop III star. However our analytical criteria for the jet breakout are also useful for the binary case.

Question: What is the detection rate of Pop III GRBs by X-ray?

Kuninito Ioka: 1/yr at most. Please see de Souza, Yoshida \& Ioka (2011) for details. 